\title{
Transcriptome analysis of carnation (Dianthus caryophyllus L.) based on next-generation sequencing technology
}

Koji Tanase ${ }^{1 *}$, Chikako Nishitani ${ }^{2}$, Hideki Hirakawa ${ }^{3}$, Sachiko Isobe ${ }^{3}$, Satoshi Tabata ${ }^{3}$, Akemi Ohmiya ${ }^{1}$ and Takashi Onozaki ${ }^{1}$

\begin{abstract}
Background: Carnation (Dianthus caryophyllus L.), in the family Caryophyllaceae, can be found in a wide range of colors and is a model system for studies of flower senescence. In addition, it is one of the most important flowers in the global floriculture industry. However, few genomics resources, such as sequences and markers are available for carnation or other members of the Caryophyllaceae. To increase our understanding of the genetic control of important characters in carnation, we generated an expressed sequence tag (EST) database for a carnation cultivar important in horticulture by high-throughput sequencing using 454 pyrosequencing technology.

Results: We constructed a normalized cDNA library and a 3'-UTR library of carnation, obtaining a total of 1,162,126 high-quality reads. These reads were assembled into 300,740 unigenes consisting of 37,844 contigs and 262,896 singlets. The contigs were searched against an Arabidopsis sequence database, and $61.8 \%(23,380)$ of them had at least one BLASTX hit. These contigs were also annotated with Gene Ontology (GO) and were found to cover a broad range of GO categories. Furthermore, we identified 17,362 potential simple sequence repeats (SSRS) in 14,291 of the unigenes. We focused on gene discovery in the areas of flower color and ethylene biosynthesis. Transcripts were identified for almost every gene involved in flower chlorophyll and carotenoid metabolism and in anthocyanin biosynthesis. Transcripts were also identified for every step in the ethylene biosynthesis pathway.

Conclusions: We present the first large-scale sequence data set for carnation, generated using next-generation sequencing technology. The large EST database generated from these sequences is an informative resource for identifying genes involved in various biological processes in carnation and provides an EST resource for understanding the genetic diversity of this plant.
\end{abstract}

Keywords: Carnation, Dianthus caryopyllus L., Next-generation sequencing technology, SSR, Transcriptome

\section{Background}

Carnation (Dianthus caryophyllus L.) is one of the most popular cut flowers, and hundreds of cultivars are grown around the world. Dianthus is a genus of about 300 species in the Caryophyllaceae family. Several species, including Dianthus caryophyllus, D. barbatus, D. chinensis, D. plumarius, D. superbus, and their hybrids are widely used as horticultural cultivars [1]. The many flower varieties of carnation are divided into three

\footnotetext{
* Correspondence: tanase@affrc.go.jp

${ }^{1}$ National Institute of Floricultural Sciences, National Agriculture and Food Research Organization, Fujimoto 2-1, Tsukuba, Ibaraki 305-8519, Japan Full list of author information is available at the end of the article
}

groups (standards, sprays, and pot carnations) based on plant form, flower size, and flower shape. Standards have a single large flower per stem, whereas sprays have a larger number of smaller flowers; both types are used for cut flowers [2]. Pot carnation is a dwarf with many small flowers that is used as a potted plant. Most carnation cultivars are diploid $(2 n=2 x=30)$, although some species of Dianthus are tetraploid or hexaploid [3-6]. According to the Plant C-values Database (http://data. kew.org/cvalues/), the total genome size (C-value) in carnation is $613 \mathrm{Mb}(1.23 \mathrm{pg} / 2 \mathrm{C})$, which is four times that of the model plant Arabidopsis $(0.30 \mathrm{pg} / 2 \mathrm{C})$ [7]. The genome of carnation is very small compared with

\section{Biomed Central}


those of other ornamental flowers, such as Antirrhinum majus (1,568 Mb), Chrysanthemum morifolium (9,384 Mb), Ipomoea nil (Pharbitis nil) (1,127 Mb), Lilium longiflorum (34,496 Mb), Petunia hybrida (1,642 Mb), Rosa hybrida $(1,127 \mathrm{Mb})$, and Tulipa gesneriana (26,093 Mb).

Carnation cultivars are developed to be highly heterozygous so as to avoid the effects of inbreeding depression [8]. Most commercially important cultivars are hybrids that are propagated vegetatively. Carnation cultivars have been bred for attractive characteristics such as flower color, flower size, fragrance, and flower longevity. Carnation cultivars have a wide range of colors, including red, yellow, white, green, and brown. In addition, some flowers show marginal variegation, flecks, or sectors [9]. Recently, transgenic carnations with blue or violet flowers have been developed by the introduction of a heterologous flavonoid 3', 5'-hydroxylase gene [10-12].

The vase life of cut flowers is one of the most important ornamental traits, because it affects consumer satisfaction and repeat purchasing. Carnation is a typical ethylene-sensitive flower [13,14], and its flower life is normally short (about 7 days) if preservatives are not used [15]. In the ethylene biosynthesis pathway, the conversion of $S$-adenosylmethionine (AdoMet) to 1aminocyclopropane-1-carboxylate (ACC) and of ACC to ethylene are catalyzed by ACC synthase (ACS) and ACC oxidase (ACO), respectively. Transgenic carnations containing an antisense $A C O$ gene exhibited low ethylene production and delayed petal senescence [16]. When the Arabidopsis etr1-1 gene, capable of conferring ethylene insensitivity, was introduced into carnation, the transgenic carnation plants had reduced ethylene sensitivity caused by suppression of $A C O$ expression, which prolonged flower life [17]. On the other hand, by repeated selection for lines with longer vase life, Onozaki et al. [18] produced two carnation cultivars (named 'Miracle Rouge' and 'Miracle Symphony') with improved vase life in which expression of three ethylene biosynthesis genes (DcACS1, DcACS2, and DcACO1) was suppressed in flowers of both cultivars, which resulted in extremely low levels of ethylene production $[18,19]$.

Expressed sequence tag (EST) sequencing is essential for functional genomics studies: it has been used to identify novel genes from a broad range of organisms and to provide an indication of gene expression levels in specific tissues. Currently, there are more than 69 million ESTs in the database (dbEST) at NCBI. Since the development of high-throughput DNA sequencing technologies, analyses using next-generation sequencers have been performed in cereals, legumes, and fruits, and large amounts of EST data have been submitted to various DNA databases. These studies have revealed that highthroughput DNA sequencing is a cost-effective approach to analyzing the ESTs of both model plants and non- model plants. Surprisingly, in Arabidopsis, at least 60 transcripts which did not exist in previous EST collections were identified by next-generation sequencing [20]. Furthermore, large-scale EST collection facilitates the design of microarrays and the high-throughput identification of simple sequence repeats (SSRs) and singlenucleotide polymorphisms (SNPs).

To identify the genes related to flower quality and important agronomic traits such as disease resistance, extensive gene expression profiling would be extremely valuable, but only 669 carnation ESTs were available on the NCBI website (http://www.ncbi.nlm.nih.gov/) at the early June 2012. Other genomics resources, such as markers and genomic sequences have yet to be developed for carnation. To improve the DNA sequence information available for carnation, we performed largescale transcriptome sequencing of carnation using a next-generation sequencer (a Roche 454 GS FLX) and obtained more than 300,000 transcripts. This work will make a significant contribution toward plant physiology, biotechnology, and molecular genetics studies in carnation.

\section{Results and discussion}

\section{EST sequencing and assembly}

To maximize the range of transcript diversity, we extracted and pooled RNA from vegetative tissues, flowers at various developmental stages, and ethylene-treated flowers of 'Francesco', a major standard-type carnation cultivar. Two libraries, a normalized cDNA library and a 3'-UTR library, were synthesized from the RNA pool, and GS FLX 454 pyrosequencing runs were performed on these libraries. We obtained data from a cDNA library that had been previously sequenced by conventional Sanger (dideoxy-based) sequencing to identify SSRs [21]. The cDNA library was synthesized from RNA of aerial part of carnation. A total of 1,435,398 reads were obtained, of which 17,988 reads $(1.25 \%)$ were obtained from Sanger sequencing (Table 1). After Cleaning (removal of adaptor sequences, poly(A) tails, etc.) of these sequences, the 454 sequencing of the normalized cDNA library generated 1,078,260 reads with an average

Table 1 Summary of carnation transcripts data

\begin{tabular}{ll}
\hline Total reads $^{\mathrm{a}}$ & 1435398 \\
Total high-quality reads & 1162126 \\
Reads in contigs & 899230 \\
Total contigs & 37844 \\
Singlets & 262896 \\
Total unique sequences (contigs plus singlets) & 300740 \\
\hline
\end{tabular}

a Includes sequences in Table 2 plus an additional 17,988 sequences obtained by Sanger sequencing of a cDNA library. 
length of 284 bp; 90,891 reads (8\%) were less than 100 bp or less (Table 2). The 454 sequencing of the 3'UTR library generated 339,150 reads with an average length of $323 \mathrm{bp}$; of these, 30,785 (9\%) were less than $100 \mathrm{bp}$ or less. These sequences resulted in a total $1,162,126$ high-quality reads (Table 1 ). After clustering and assembly, 899,230 sequences were incorporated into 37,844 contigs, leaving 262,896 singlets, for a total of 300,740 unique sequences (Table 1). The average length of the contigs was $605 \mathrm{bp}$ (range 117-3,850 bp). The 300,740 sequences were first compared with the sequences in the non-redundant NCBI database by using BLASTN. Next, for Gene Ontology (GO) classification, the contigs were annotated by searching for sequence similarities using BLASTX against Arabidopsis genes (TAIR v.7.0; www.arabidopsis.org); $62 \%$ of the contigs (23,380 sequences) had at least one BLASTX hit. The percentage similarity between the carnation sequences and those of Arabidopsis was highly dependent on the length of the query sequence, as was previously seen in Eucalyptus [22]: longer sequences gave higher percent similarity. Contigs of carnation transcripts that were longer than $117 \mathrm{bp}$ may be of good quality for similarity searches (data not shown). Recently, a number of large-scale EST data sets have been successfully constructed from non-model plants, including maize, chestnut, olive, the medicinal herb Artemisia annua, and Cucurbita pepo, by using high-throughput sequencing with the GS FLX 454 sequencer [23-26]. We compared the sequence length of these results and ours, and found no great differences; therefore, we judged our carnation transcripts data to be of sufficiently high quality for further investigation.

\section{Functional annotation}

GO has a controlled vocabulary that describes gene products in terms of their associated biological processes, cellular components, and molecular functions. We utilized the GO assignments of Arabidopsis gene models (the GO Slim classifications in TAIR) for assignment of putative functional roles to the 37,844 contigs of

Table 2 Size distribution of 454 sequencing reads after removal of adaptor sequences

\begin{tabular}{lcccccc}
\hline & \multicolumn{2}{c}{ Normalized cDNA library } & & \multicolumn{2}{c}{ 3'-UTR library } \\
\cline { 2 - 3 } \cline { 5 - 6 } Read length & Number of reads & $\%$ & & Number of reads & $\%$ \\
\hline S100 bp & 90891 & 8.4 & & 30785 & 9.1 \\
$101-250 \mathrm{bp}$ & 382543 & 35.5 & & 89507 & 26.4 \\
$251-500 \mathrm{bp}$ & 557048 & 51.7 & & 177269 & 52.3 \\
$501-750 \mathrm{bp}$ & 47743 & 4.4 & & 41584 & 12.3 \\
$\mathbf{2 7 5 1} \mathrm{bp}$ & 35 & 0.0 & & 5 & 0.0 \\
Total & 1078260 & 100 & 339150 & 100 \\
\hline
\end{tabular}

carnation. The top GO category matches for 17,584 genes of Arabidopsis were assigned to 23,380 (61.8\%) of the contigs $\left(\mathrm{E}\right.$-value $\leq 10^{-5}$ ). These genes covered a broad range of GO categories (Figure 1), and some genes were assigned to more than one category. The most common assignments in the Biological Process category were protein metabolism (27\%), transport (12\%), transcription (12\%), cell organization and biogenesis (11\%), developmental processes $(9 \%)$, and response to abiotic or biotic stimulus (8\%). In the Cellular Component category, the largest classes were chloroplast (27\%), nucleus (26\%), mitochondria (12\%), and plastid (11\%). In the Molecular Function category, the most common assignments were hydrolase activity (19\%), transferase activity (13\%), protein binding (12\%), DNA or RNA binding (11\%), transcription factor activity (11\%), and kinase activity (9\%). The proportions of genes assigned to each GO category were very similar to those found in the genome annotation of Arabidopsis. The GO assignment analysis reinforces our assumption that a broad diversity of genes was sampled by using the selected tissues. Overall, these results of sequencing and functional annotation indicate that the large-scale sequencing technology is an efficient method for the transcriptome analysis of plants, especially those currently lacking other genomics tools.

\section{SSR marker discovery}

SSRs are useful as random markers for population genetics research. To facilitate population genetics analysis and genetic mapping studies in carnation, we identified SSR motifs in our 454 data set. The reads from the 454 runs on the normalized cDNA library and the 3'-UTR library were used for EST-SSR discovery. The reads from conventional Sanger sequencing were not used for SSRs discovery in this study because SSR motifs from these reads were identified in a previous study [21]. Of the $1,417,410$ sequences from 454 sequencing, 1,041,854 were incorporated into 109,951 contigs, leaving 375,556 singlets, for a total of 485,507 unique sequences after cleaning (removal of adaptors, poly(A) tail, etc), clustering, and assembly. A screen for the presence of SSRs was performed on this data set using the MISA program (http://pgrc.ipk-gatersleben.de/misa/). A search for di-, tri-, tetra-, and pentanucleotide repeats identified a total of 17,362 potential SSRs in 14,291 unigenes; that is, approximately $3 \%$ of the unigenes contained at least one of the SSR motifs included in the search (Table 3). This percentage was among the lower values seen for other species, in which approximately 3\% to $20 \%$ of ESTs contained putative SSR motifs [23,27-29]. Finally, a total of 4,177 SSR primer pairs were designed from these unigenes using the MIRA3.2. program (http:// mira-assembler.sourceforge.net/) (data not shown). Although further studies are needed to investigate and 


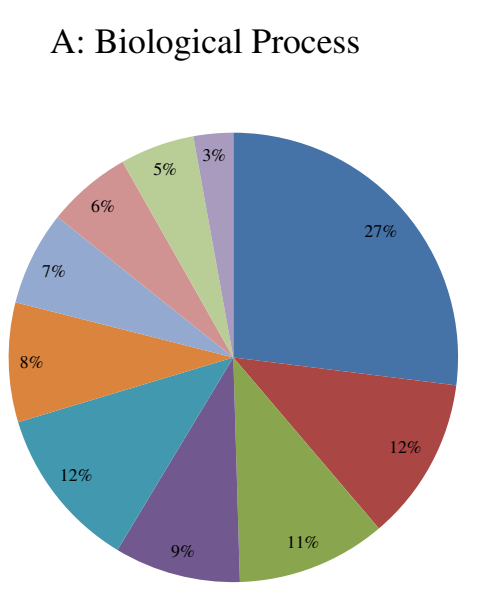

B: Cellular Component

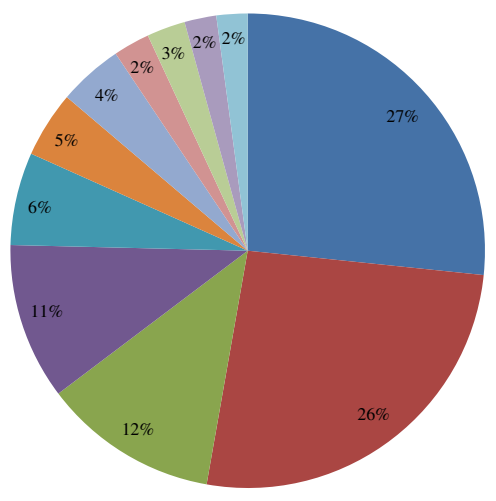

C: Molecular Function

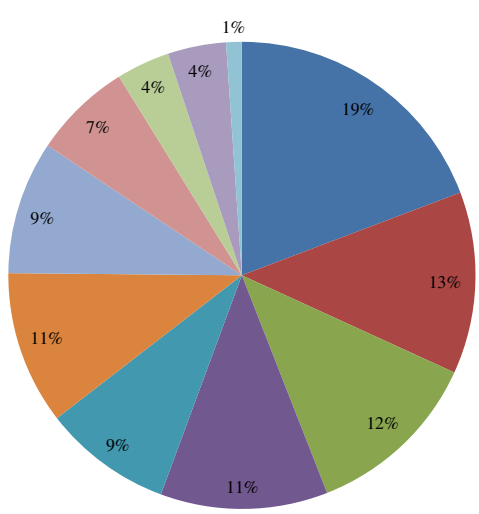

- protein metabolism

transport

cell organization and biogenesis

ndevelopmental processes

- transcription

response to abiotic or biotic stimulus

response to stress

- signal transduction

electron transport or energy pathways

- DNA or RNAmetabolism

chloroplast
nucleus
mitochondria
plastid
$\square$ ribosome
cytosol
plasma membrane
cell wall
extracellular
ER

- hydrolase activity

- transferase activity

- protein binding

- DNA or RNA binding

kinase activity

transcription factor activity

transporter activity

nucleotide binding

nucleic acid binding

structural molecule activity

receptor binding or activity

Figure 1 Classification of transcripts into functional categories according to Arabidopsis Gene Ontology. A: Biological Process, B: Cellular Component, C: Molecular Function. In the Biological Process category, "other biological processes", "other metabolic processes", and "other cellular processes" were omitted. In the Cellular Component category, "other cytoplasmic components", "other cellular components", "other membranes", and "other intracellular components" were omitted. In the Molecular Function category, "Other molecular functions", "other binding", and "other enzyme activity" were omitted. 
Table 3 Number of di-, tri-, tetra-, and pentanucleotide simple sequence repeats (SSRs) identified in $1,417,410$ reads obtained by 454 sequencing

\begin{tabular}{|c|c|c|}
\hline Dinucleotide repeat & $\begin{array}{l}\text { Number of } \\
\text { dinucleotide SSRs }\end{array}$ & $\%$ \\
\hline AC/GT & 150 & 15 \\
\hline $\mathrm{AG} / \mathrm{CT}$ & 321 & 33 \\
\hline AT/TA & 170 & 17 \\
\hline $\mathrm{CA} / \mathrm{TG}$ & 81 & 8 \\
\hline GA/TC & 250 & 26 \\
\hline Total & 972 & 100 \\
\hline Trinucleotide repeat & $\begin{array}{l}\text { Number of } \\
\text { trinucleotide SSRs }\end{array}$ & $\%$ \\
\hline $\mathrm{AAG} / \mathrm{CT}$ & 616 & 6 \\
\hline AAT/ATT & 652 & 7 \\
\hline $\mathrm{AGA} / \mathrm{TCT}$ & 787 & 8 \\
\hline ATC/GAT & 614 & 6 \\
\hline $\mathrm{CAA} / \mathrm{TG}$ & 811 & 8 \\
\hline GAA/TTC & 871 & 9 \\
\hline TCA/TGA & 768 & 8 \\
\hline Other trinucleotide repeats & 4613 & 47 \\
\hline \multicolumn{3}{|l|}{ ( $<5 \%$ of each one) } \\
\hline Total & 9732 & 100 \\
\hline Tetranucleotide repeat & $\begin{array}{l}\text { Number of } \\
\text { tetranucleotide SSRs }\end{array}$ & $\%$ \\
\hline AAAT/ATTT & 176 & 7 \\
\hline AATC/GATT & 165 & 7 \\
\hline ATTA/TAAT & 145 & 6 \\
\hline ATTG/CAAT & 121 & 5 \\
\hline TCAA/TTGA & 287 & 12 \\
\hline Other tetranucleotide repeats & 1498 & 63 \\
\hline \multicolumn{3}{|l|}{ ( $<5 \%$ of each one) } \\
\hline Total & 2392 & 100 \\
\hline Pentanucleotide repeat & $\begin{array}{l}\text { Number of } \\
\text { pentanucleotide SSRs }\end{array}$ & $\%$ \\
\hline AAAAT/ATTTT & 401 & 9 \\
\hline Other pentanucleotide repeats & 3865 & 91 \\
\hline \multicolumn{3}{|l|}{ ( $<5 \%$ of each one) } \\
\hline Total & 4266 & 100 \\
\hline
\end{tabular}

select for markers that show polymorphism, these data will provide powerful tools for the identification of markers linked to beneficial characters.

Very few genetic markers for horticulturally important characters in the major ornamentals, including carnation, have been identified [30]. To our knowledge, only a few studies have reported SSR marker development in carnation [21,31-33]. Smulders et al. [32,33] developed 8 SSR markers from the EMBL database and evaluated the genetic diversity in Dianthus species. These SSR markers were also used for constructing a genetic linkage map of carnation [34]. Kimura et al. [31] developed a set of 13 SSR markers and demonstrated their usefulness for genetic identification and hybridity confirmation of interspecific crosses in Dianthus species. Recently, a comprehensive set of 4,323 SSR primer pairs, representing 178 unique marker loci in 16 linkage groups, was developed and experimentally validated for carnation [21]; one of these loci was identified as a quantitative trait locus for carnation bacterial wilt resistance. In general, SSRs derived from ESTs are tightly linked with functional genes that may control useful characters. Furthermore, SSR markers can contribute to the construction of genetic linkage maps, genetic identification, and parentage analysis in Dianthus species.

\section{Transcripts related to flower color}

Red and yellow petal colors in higher plants are generally produced by anthocyanins and carotenoids, respectively, but species belonging to the order Caryophyllales show unique pigment composition in their flowers. In most of the Caryophyllales, red and yellow petal colors are derived from betalains; most of them accumulate neither anthocyanin nor carotenoids in their flowers. Carnation is an exception in that it accumulates anthocyanins and can express red and pink colors. The yellow petal color of carnation cultivars is derived from chalcone, a yellow flavonoid, rather than from carotenoids. Although chlorophylls are generally absent from the flowers of most plants, some carnation cultivars accumulate chlorophylls in their petals and have a green flower phenotype. It will therefore be interesting to investigate the expression of genes involved in the metabolism of these pigments in members of the Caryophyllales. The carnation EST database will provide useful information for future studies at the molecular level.

\section{Carotenoid and chlorophyll metabolism}

Carotenoids are isoprenoid compounds synthesized from isopentenyl diphosphate (IPP), a five-carbon isoprene unit. Because IPP is a precursor of various physiologically important compounds such as chlorophyll, tocopherol, gibberellin, and cytokinin [35], genes encoding isoprenoid biosynthesis enzymes might be expressed throughout the plant body. In Arabidopsis, all of the genes for isoprenoid biosynthesis are expressed in both flowers and leaves (TAIR: http://www.arabidopsis.org/). However, among the genes upstream of IPP, only deoxyxyllulose 5phosphate synthase (DXS) was found in the carnation EST database. On the other hand, we found transcripts corresponding to most enzymes functioning 
downstream of IPP and leading to the synthesis of carotenoids. The database contained more than one transcript each for isopentenyl pyrophosphate isomerase, geranylgeranyl diphosphate synthase, phytoene synthase, phytoene desaturase, $\zeta$-carotene desaturase, carotenoid isomerase, lycopene $\beta$-cyclase, lycopene $\epsilon$ cyclase, $\beta$-ring hydroxylase, $\epsilon$-ring hydroxylase, and violaxanthin de-epoxidase (Figure 2). Only one transcript was found for zeaxanthin epoxidase. Galpaz et al. [36] reported that multiple homologs of geranylgeranyl diphosphate synthase, phytoene synthase, $\zeta$ carotene desaturase, and $\beta$-ring hydroxylase are present in tomato and expressed in a tissue-specific manner. It is of great interest to learn the tissue specificity of the multiple homologs of the carotenoid

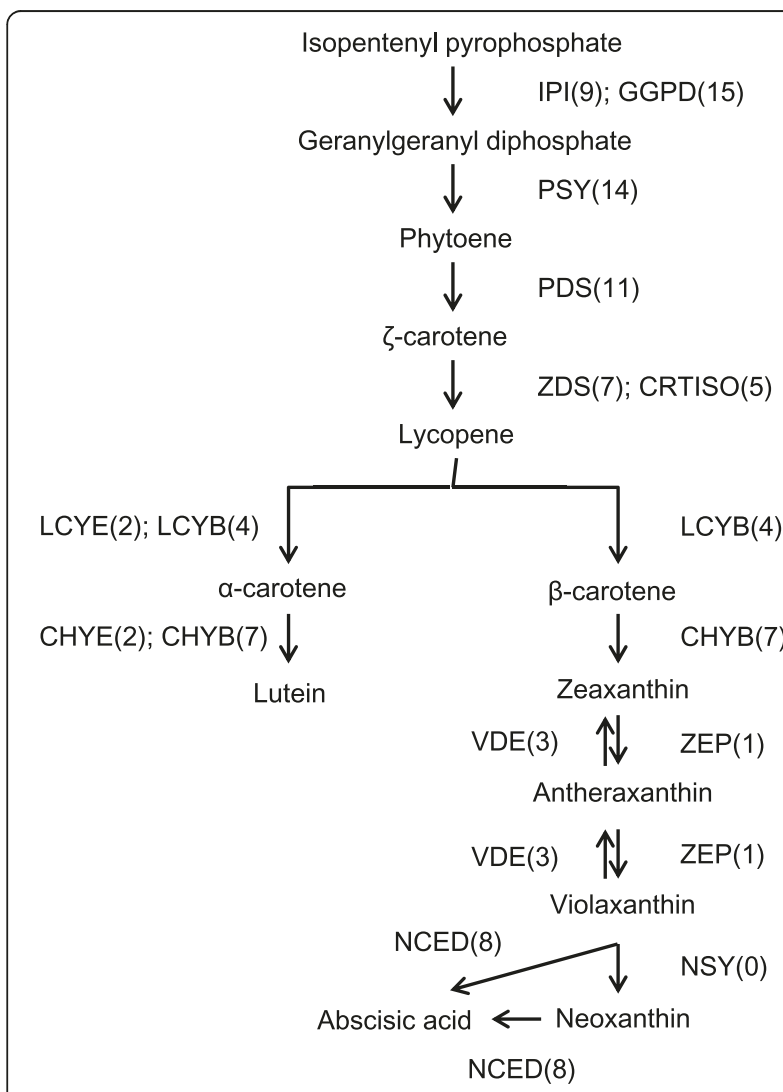

Figure 2 Distribution of carnation transcripts in the carotenoid biosynthesis pathway. Previously published sequences in GenBank belonging to the carotenoid biosynthesis pathway were used in BLAST searches to identify genes in the carnation EST database. Each enzyme name is followed in parentheses by the number of contigs homologous to gene families encoding this enzyme. IPI, isopentenyl pyrophosphate isomerase; GGDP, geranylgeranyl diphosphate synthase; PSY, phytoene synthase; PDS, phytoene desaturase; ZDS, Z-carotene desaturase; LCYB, lycopene $\beta$-cyclase; LCYE, lycopene $\epsilon$-cyclase; CHYB, $\beta$-ring hydroxylase; CHYE, $\epsilon$-ring hydroxylase; ZEP, zeaxanthin epoxidase; VDE, violaxanthin deepoxidase; CRTISO, carotenoid isomerase; NSY, neoxanthin synthase: NCED, 9-cis-epoxycarotenoid dioxygenase. biosynthesis genes found in the carnation transcripts database.

Carotenoid catabolism produces diverse apocarotenoid compounds that are essential for plant growth and reproduction [37]. One category of these compounds (abscisic acid and strigolactone) is categorized as a plant hormone, and the others provide fruits and flowers with aromas and colors for attracting pollinators and seed dispersers. Such bioactive apocarotenoids are produced when carotenoids are cleaved by carotenoid cleavage dioxygenase (CCD). Analysis of the genome sequence of Arabidopsis led to the definition of nine clades of dioxygenases [38]. Five of these, the 9-cis epoxycarotenoid dioxygenases (NCEDs; NCED2, NCED3, NCED5, NCED6, and NCED9) are involved in the synthesis of the plant hormone abscisic acid. The remaining four are involved in the synthesis of the plant hormone strigolactone (CCD7 and CCD8), in aroma formation (CCD1), and in the regulation of carotenoid content in the flower (CCD4). The carnation transcripts database contained sequences corresponding to two types of NCEDs, which showed high sequence similarity to NCED2 and NCED5, and one type of CCD, which showed high sequence similarity to CCD1.

The chlorophyll metabolic pathway can be classified into three distinct phases: chlorophyll biosynthesis, the chlorophyll cycle, and chlorophyll degradation [39]. Numerous enzymes function in these processes, and most of the genes encoding these enzymes were represented in the carnation transcripts database. Among the 14 enzymes involved in chlorophyll biosynthesis, transcripts corresponding to 12 enzymes were found in the database; the only ones not represented were uroporphyrinogen III synthase and Mgproto IX monomethylester cyclase (Figure 3). Transcripts for chlorophyllide $a$ oxygenase and chlorophyll $b$ reductase, both of which are involved in the chlorophyll cycle, were found in the carnation database. Among the enzymes involved in chlorophyll degradation, transcripts corresponding to pheophorbide $a$ oxygenase were found, but chlorophyllase, pheophytinase, and red chlorophyll catabolite reductase (RCCR) were not.

Chlorophylls and carotenoids are essential pigments that play important roles in photosynthesis. In 'Francesco' carnation, high levels of these pigments were found in the leaves but not in the flowers (data not shown). Thus, the transcripts related to carotenoid and chlorophyll biosynthesis might have been derived from leaves. On the other hand, chlorophyll degradation is generally activated during leaf senescence. The absence of transcripts for some chlorophyll degrading enzymes might be explained by the fact that RNA was obtained from flowers and developing leaves but not from senescent leaves. 


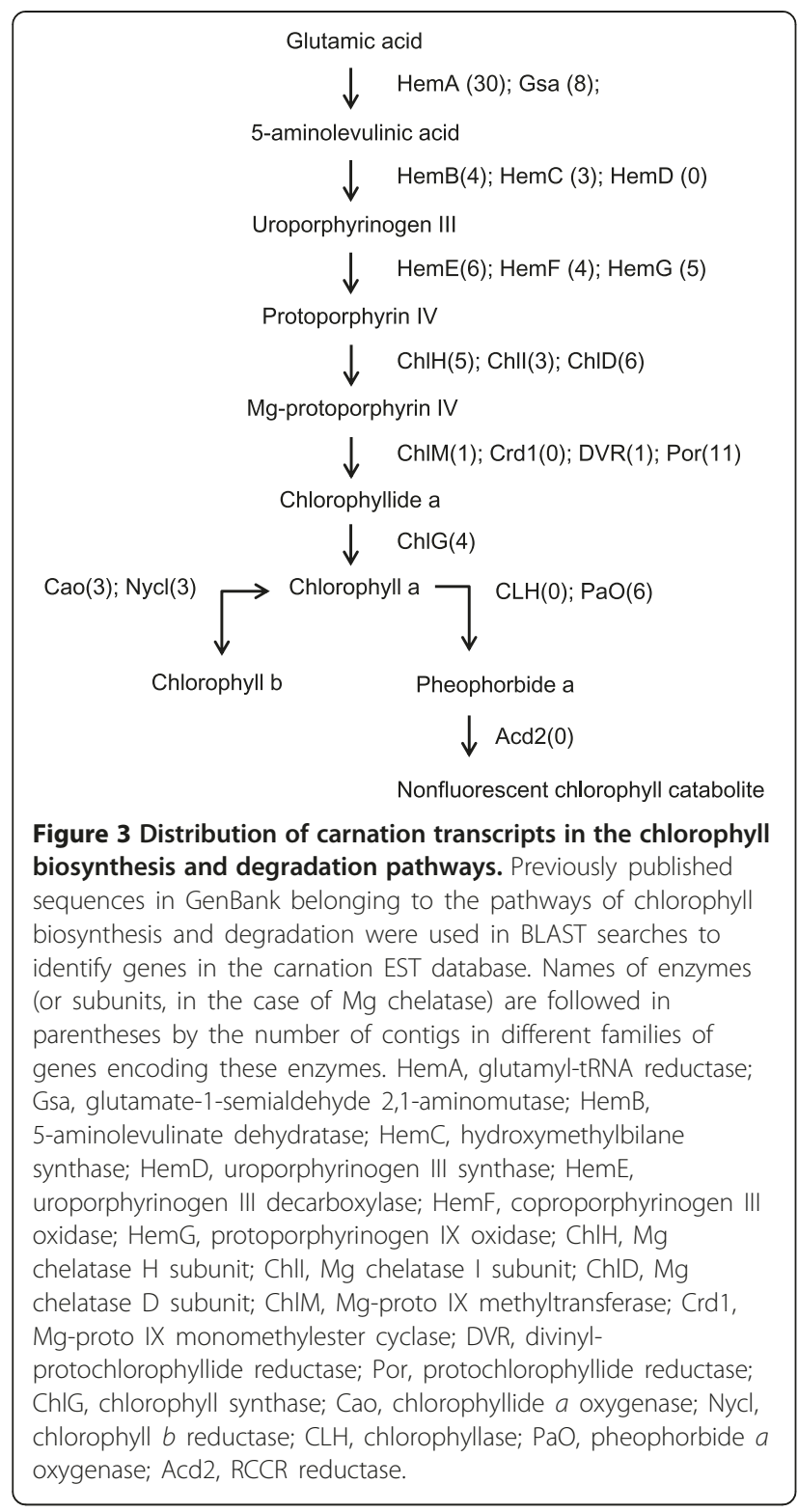

\section{Anthocyanin biosynthesis}

Anthocyanins are widely occurring colorants of fruits and flowers. Anthocyanidins, which are precursors of anthocyanins, are classified into six types: cyanidin, pelargonidin, peonidin, delphinidin, petunidin, and malvidin. Carnation flowers contain mainly pelargonidinand cyanidin-type anthocyanidins [40-43]. Enzymes functioning in the anthocyanidin biosynthesis pathway have been well studied in many plants $[12,44]$. ESTs of all the enzymes involved in the anthocyanidin biosynthesis pathway (from phenylalanine to anthocyanidin) were present in the carnation transcripts database (Figure 4). Every enzyme in the pathway was represented by multiple transcripts except for 4-coumaroyl CoA ligase and anthocyanidin synthase, each of which was represented by a single EST.

Anthocyanidins are modified by glycosylation and acylation, to form anthocyanins. These modifications play important roles in changing flower color, increasing water solubility, and enhancing pigment stability. Recently, two types of glucosyltransferase have been identified and characterized in carnation $[45,46]$. Here, we found multiple transcripts encoding anthocyanidin glucosyltransferase and

Phenylalanine

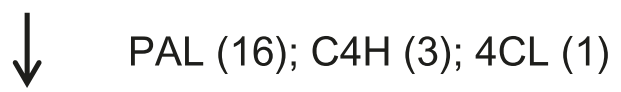

4-coumaroyl-CoA<smiles>CCCC[AsH3]</smiles>

Chalcone<smiles>[13CH]=C[Mg]</smiles>

Flavanone

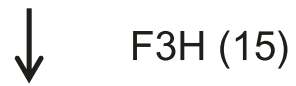

Dihydrokaempferol<smiles>CCCCCCCC</smiles>

Leucoanthocyanin<smiles>CCCCCCCCC</smiles>

Anthocyanidin

$$
\downarrow \quad \text { GT (3); AT (4) }
$$

\section{Anthocyanin}

Figure 4 Distribution of carnation transcripts in the anthocyanin (flavonoid) biosynthesis pathway. Previously published sequences in GenBank belonging to the flavonoid biosynthesis pathway were used in BLAST searches to identify genes in the carnation EST database. Each enzyme name is followed in parentheses by the number of contigs homologous to gene families encoding this enzyme. PAL, phenylalanine ammonia-lyase; $\mathrm{C} 4 \mathrm{H}$, cinnamate 4-hydroxylase; 4CL, 4-coumaroyl:CoA ligase; CHS, chalcone synthase; $\mathrm{CH}$, chalcone isomerase; $\mathrm{F} 3 \mathrm{H}$, flavanone 3-hydroxylase; DFR, dihydroflavonol 4-reductase; ANS, anthocyanidin synthase; GT, anthocyanidin glucosyltransferase; AT, anthocyanin acyltransferase. 


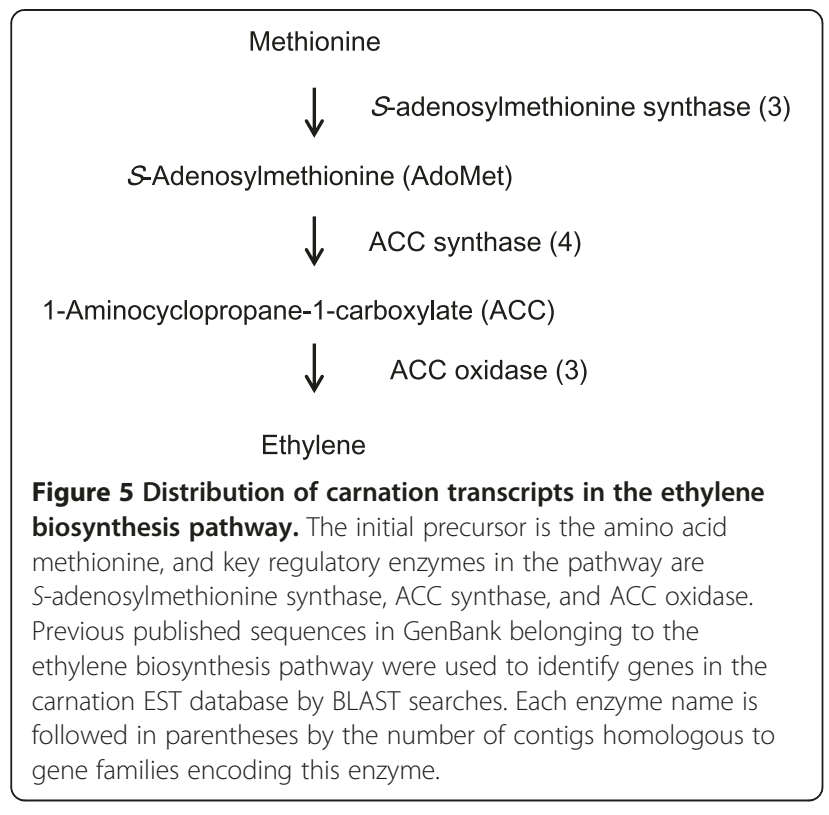

anthocyanin acyltransferase in the database. Thus, the carnation transcripts database will contribute to further investigations into the diversity of anthocyanin modification mechanisms.

\section{Betalain biosynthesis}

Although the betalain biosynthesis pathway is poorly understood, several enzymes involved in this pathway have been identified and characterized [12,47]. Among them, an transcripts encoding dihydroxyphenylalanine (DOPA) dioxygenase was found in the transcripts database; further investigation will be necessary to verify if a part of the betalain biosynthesis pathway is active in the carnation flower.

\section{Ethylene biosynthesis and signaling}

Ethylene is a gaseous plant hormone with many important roles in growth and development [48], and is involved in flower senescence in many species [14]. The deterioration of the corolla in these species is accelerated by exogenous ethylene, and senescence is accompanied by an increase in endogenous ethylene biosynthesis [49]. The regulation of senescence in carnation, which is one of the most ethylene-sensitive flowers, has been investigated through the study of the expression of genes related to ethylene biosynthesis. In many plant species, including carnation, the pathway of ethylene biosynthesis is well characterized, having $S$ adenosylmethionine (AdoMet) as a starting compound and 1-aminocyclopropane-1-carboxylate (ACC) as an intermediate [50]. The conversion of methionine to AdoMet is catalyzed by $S$-adenosylmethionine synthase, the conversion of AdoMet to ACC by ACS, and the conversion of ACC to ethylene by ACO [51]. The carnation EST database contained multiple ESTs encoding each of these three ethylene biosynthesis enzymes (Figure 5). We cloned a cDNA encoding a novel $A C O$ gene from the transcripts database constructed in this study (data not shown). Other carnation cDNA clones representing $S$-adenosylmethionine synthase, ACS, and ACO had high sequence similarity to those that have already been reported (Accession No. P24260, M66619, AF049138, AF049137, M62380). Additionally, we found ESTs corresponding to most ethylene signal pathway genes (Table 4). The database contained more than one EST each for ethylene receptors, EILs (ETHYLENE INSENSITIVE3-like) and ERFS (Ethylene-responsive-element-binding factor). The members of these families are involved in the regulating various biological processes such as autocatalytic ethylene production, senescence, and various responses to stress through the ethylene perception [52]. Understanding the functions of these genes will help our understanding of regulation of ethylene dependent flower senescence in carnation.

During flower senescence in carnation, a burst of ethylene production occurring in the gynoecium is followed by ethylene delivered to the petals, though the identity of the trigger signal molecule is still unknown. Autocatalytic ethylene production is induced by the signal, which in turn initiates downstream events in the senescence process such as lipid peroxidation and proteolytic activity $[53,54]$. Therefore, there is much interest in the regulation of senescence by the expression of genes related to ethylene biosynthesis. In many ethylene-sensitive flowers, ACS and ACO are key steps in ethylene production, and transcript levels of the corresponding genes are rapidly upregulated at the ethylene burst stage [49,50,54]. These findings suggest that $A C S$ and $A C O$ gene expression is transcriptionally regulated in carnation.

As mentioned in the Background section, the improved cultivars 'Miracle Rouge' and 'Miracle Symphony' have very long flower life (average 18 days) and show much lower ethylene production than normal cultivars [18]. In these improved cultivars, the expression levels of DcACS1, DcACS2 and DcACO1 were low throughout the experimental period [19], but sequencing of genomic DNA did not detect any mutations in these genes (Tanase, unpublished). On the other hand, custom-

\section{Table 4 Distribution of carnation transcripts in the} ethylene signaling pathway

\begin{tabular}{ll}
\hline Gene name & Number of contigs \\
\hline Ethylene receptor & 6 \\
(ETHYLENE INSENSITIVE3-like) EIL & 3 \\
Ethylene-responsive-element-binding factor (ERF) & 4 \\
\hline
\end{tabular}


made cDNA microarrays of carnation showed that some transcripts encoding transcription factors, including EIN3-like (EIL) transcription factors, a putative MYB-like protein, a zinc finger protein, a MYC-type protein, and MADS-box proteins, were upregulated during flower senescence [52]. In tomato, the MADS-box protein RIN (ripening inhibitor) regulates fruit ripening through direct activation of LeACS2 [55,56]. Other transcription factors such as TOMATO AGAMOUS-LIKE 1 MADS-box protein and tomato HD-Zip homeobox protein, which regulate fruit ripening, probably control the expression of ethylene biosynthesis genes $[57,58]$. Our carnation database included many contigs related to transcription factor activity (11\% of the Molecular Function ESTs) in the GO function analysis (Figure 1). Thus, the carnation transcripts database will contribute to further investigations into the regulation of ethylene biosynthesis and senescence programs in flowers.

\section{Conclusions}

In this study, an EST database was developed to enable broad characterization of the carnation transcriptome. We detected 17,362 potential simple sequence repeats (SSRs) in 14,291 unigenes and identified transcripts corresponding to genes associated with carotenoid biosynthesis, chlorophyll biosynthesis and degradation, anthocyanin (flavonoid) biosynthesis, and ethylene biosynthesis and signaling. This collection of transcripts from carnation will be useful for the annotation of the forthcoming carnation genome sequence and provide a remarkable resource for genomics studies in Caryophyllaceae.

\section{Methods}

\section{Plant materials and RNA extraction}

Carnation (Dianthus caryophyllus L.) cultivar 'Francesco' was grown under natural daylight conditions in a greenhouse as described previously [15]. Each tissue was harvested from three plants. The following plant tissues were used: flower bud, flower (day 0 [full open flower], 3 days after full open, 8 days after full open, 4-h ethylene

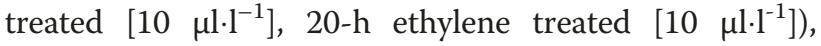
young and adult leaves, and stem (with shoot apex). Flowers contained sepals, petals, stamens and pistils. Tissues were immediately frozen in liquid nitrogen and stored at $-80^{\circ} \mathrm{C}$. Total RNA was extracted using the RNeasy Plant Mini Kit (Qiagen, Hilden, Germany). RNA concentration was estimated using an ND-1000 spectrophotometer (NanoDrop) (Thermo Scientific, Wilmington, DE, USA) and RNA integrity was evaluated using an Agilent 2100 Bioanalyzer (Agilent Technologies, Santa Clara, CA, USA).

\section{cDNA library construction for $\mathbf{4 5 4}$ sequencing}

For 454 sequencing, we made a normalized cDNA library and a 3' cDNA library in cooperation with Takara Bio (Otsu, Shiga, Japan). RNA isolated from each tissue was combined in equal proportions in a single pool in an attempt to maximize the diversity of transcriptional units sampled. The Clontech SMART system (Clontech, Mountain View, CA, USA) was used for cDNA synthesis from the total RNA.

To construct the normalized cDNA library, the cDNA was normalized by digestion with a duplexspecific nuclease. The normalized cDNA was amplified under the following conditions: $95^{\circ} \mathrm{C}$ for $20 \mathrm{~s}$, followed by 25 cycles of $95^{\circ} \mathrm{C}$ for $5 \mathrm{~s}$ and $68^{\circ} \mathrm{C}$ for $8 \mathrm{~min}$. The PCR primers were as follows: TD-5-P2 primer, 5'-GAGTGGCCATTACGGCCGGG-3'; biotinylated $\left(\mathrm{T}_{18}\right) \mathrm{VN}$ B-adaptor oligo, 5'-biotinCCTATCCCCTGTGTGCCTTGGCAGTCTCAGTTTT TTTTTTTTTTTTTTVN-3'. After purification, the quantity of amplified cDNA was estimated using an ND-1000 spectrophotometer (NanoDrop) and the quality was evaluated using an Agilent 2100 Bioanalyzer. Approximately $5 \mu \mathrm{g}$ of amplified cDNA was sheared into small fragments about 800 bp in length using an Acoustic Solubilizer (Covaris, Woburn, MA, USA). The cDNA library was constructed according to the manufacturer's instructions in the Roche GS FLX Titanium General Library Preparation Method Manual.

For the 3' cDNA library, we used the modified method of Eveland et al. (2008) [59]. Approximately $10 \mu \mathrm{g}$ of amplified cDNA was sheared into small fragments about $800 \mathrm{bp}$ in length with an Acoustic Solubilizer (Covaris). The cDNA fragments were selected by size, 400$1000 \mathrm{bp}$, using gel-cut and eluting them. The 3' ends of the fragments were purified by using streptavidin-coated magnetic beads. Titanium A-adaptors (Roche, Basel, Switzerland) were ligated to the purified 3' fragments, and the single-stranded 3' cDNA was treated with $100 \mathrm{mM} \mathrm{NaOH}$, neutralized, and purified. The quality of the 3' cDNA library was assessed as described above for the normalized cDNA library. The 454 sequencing was performed according to the manufacturer's instructions in the Roche GS FLX Titanium Sequencing Method Manual.

To construct the cDNA library for Sanger sequencing, poly(A) ${ }^{+}$RNA from aerial part of carnation plant was purified using Oligotex-dT30 Super (Nippon Roche, Tokyo, Japan), and cDNA was synthesized by using a cDNA synthesis kit (Agilent Technologies) according to the manufacturer's instructions. The size selection of cDNA and cloning into a pBluescript II $\mathrm{SK}^{-}$plasmid were performed as previously described [60]. For generation of ESTs, plasmid DNAs were prepared from the colonies and sequenced 
using a BigDye Terminator Cycle Sequencing Ready Reaction Kit (Applied Biosystems, Foster City, CA, USA). The reaction mixtures were run on an automated DNA sequencer (ABI PRISM 3730; Applied Biosystems).

\section{Data analysis}

Both the 454 sequences and Sanger sequences were trimmed of adaptor and low-quality sequence regions. All sequences were assembled and annotated by BLASTN searches of the NCBI database.

dditionally, the non-redundant set of consensus cDNA sequences represented by two or more reads $(37,844$ sequences) was annotated by BLAST searches of Arabidopsis cDNA databases. Functional classifications of these sequences were based on GO terms from the GO Slim classification in TAIR (www.arabidopsis.org).

\section{Accession numbers}

Assembled transcripts of Carnation (Dianthus caryophyllus L.) were submitted to the Mass Submission System of DDBJ with the accession numbers FX296474 to FX334317.

\section{Detection of SSR markers}

All of the assembled sequences from the 454 reads were used for detection of SSRs. SSRs in the total unique putative transcripts were detected by using the MISA program (http://pgrc.ipk-gatersleben.de/misa/), which accepts FASTA-formatted sequence files. Sequences containing di-, tri-, tetra-, and pentanucleotide repeats were selected.

\section{Competing interests}

The authors declare that they have no competing interests.

\section{Acknowledgements}

This study was supported by a grant from the project "Development of Innovative Crops through the Molecular Analysis of Useful Genes" of the National Agriculture and Food Research Organization (NARO), Japan.

\section{Author details \\ ${ }^{1}$ National Institute of Floricultural Sciences, National Agriculture and Food Research Organization, Fujimoto 2-1, Tsukuba, Ibaraki 305-8519, Japan. ${ }^{2}$ National Institute of Fruit Tree Science, National Agriculture and Food Research Organization, Fujimoto 2-1, Tsukuba, Ibaraki 8605, Japan. ${ }^{3}$ Kazusa DNA Research Institute, 2-6-7 Kazusa-kamatari, Kisarazu, Chiba 292-0818, Japan.}

\section{Authors' contributions}

KT prepared the CDNA libraries for 454 sequencing. SI and ST performed the conventional Sanger sequencing. $\mathrm{HH}, \mathrm{SI}$, and ST selected the SSRs and designed primer pairs. $\mathrm{KT}$ and $\mathrm{CN}$ performed the bioinformatics analysis. $\mathrm{KT}$ is the main coordinator of the carnation EST project and participated in the conception of the research together with $\mathrm{AO}$ and TO. KT was primarily responsible for drafting the manuscript. All authors read and approved the final manuscript.

Received: 6 January 2012 Accepted: 2 July 2012

Published: 2 July 2012

\section{References}

1. Fu XP, Ning GG, Gao LP, Bao MZ: Genetic diversity of Dianthus accessions as assessed using two molecular marker systems (SRAPs and ISSRs) and morphological traits. Sci Hort 2008, 117:263-270.
2. Vainstein A, Hillel J, Lavi U, Tzuri G: Assessment of genetic relatedness in carnation by DNA fingerprint analysis. Euphytica 1991, 56:225-229.

3. Ushio A, Onozaki T, Shibata M: Estimation of polyploidy levels in Dianthus germplasms by flowcytometry. Bull Natl Inst Flor Sci 2002, 2:21-26. In Japanese with English summary.

4. Yagi M, Fujita Y, Yoshimura T, Onozaki T: Comprehensive estimation of polyploidy level in carnation cultivars by flow cytometry. Bull Natl Inst Flor Sci 2007, 7:9-16. In Japanese with English summary.

5. Yagi M, Kimura T, Yamamoto T, Onozaki T: Estimation of ploidy levels and breeding backgrounds in pot carnation cultivars using flow cytometry and SSR markers. J Japan Soc Hort Sci 2009, 78:335-343.

6. Itoh A, Takeda T, Tsukamoto Y, Tomino K: Genus Dianthus. In The Grand Dictionary of Horticulture 'Compact version' vol 2 (In Japanese). Edited by Tsukamoto Y. Tokyo: Shogakukan; 1994:1671-1678. in Roman.

7. Arumuganathan K, Earle ED: Nuclear DNA content of some important plant species. Plant Mol Biol Rep 1991, 9:208-221.

8. Sato S, Katoh N, Yoshida H, Iwai S, Hagimori M: Production of doubled haploid plants of carnation (Dianthus caryophyllus L.) by pseudofertilized ovule culture. Sci Hort 2000, 83:301-310.

9. Okamura M, Yasuno N, Ohtsuka M, Tanaka A, Shikazono N, Hase Y: Wide variety of flower-color and -shape mutants regenerated from leaf cultures irradiated with ion beams. Nucl Instrum Methods Phys Res B 2003, 206:574-578.

10. Fukui $Y$, Tanaka $Y$, Kusumi T, Iwashita T, Nomoto $K$ : A rationale for the shift in colour towards blue in transgenic carnation flowers expressing the flavonoid 3', 5'-hydroxylase gene. Phytochemistry 2003, 63:15-23.

11. Tanaka Y, Fukui Y, Fukuchi-Mizutani M, Holton TA, Higgins E, Kusumi T: Molecular cloning and characterization of Rosa hybrida dihydroflavonol 4-reductase gene. Plant Cell Physiol 1995, 36:1023-1031.

12. Tanaka Y, Sasaki N, Ohmiya A: Biosynthesis of plant pigments: anthocyanins, betalains and carotenoids. Plant J 2008, 54:733-749.

13. Brown KM: Ethylene and abscission. Physiol Plant 1997, 100:567-576.

14. Woltering EJ, van Doorn WG: Role of ethylene in senescence of petals: Morphological and taxonomical relationship. J Exp Bot 1988, 39:1605-1616.

15. Onozaki T, lkeda $H$, Yamaguchi T: Genetic improvement of vase life of carnation flowers by crossing and selection. Sci Hort 2001, 87:107-120.

16. Savin KW, Baudinette SC, Graham MW, Michael MZ, Nugent GD, Lu CY, Chandler SF, Cornish EC: Antisense ACC oxidase RNA delays carnation petal senescence. Hortscience 1995, 30:970-972.

17. Bovy AG, Angenent GC, Dons HJM, van Altvorst AC: Heterologous expression of the Arabidopsis etr1-1 allele inhibits the senescence of carnation flowers. Mol Breed 1999, 5:301-308.

18. Onozaki T, Ikeda H, Shibata M, Yagi M, Yamaguchi T, Amano M: Breeding and characteristics of carnation Norin No. 1 'Miracle Rouge' and No. 2 'Miracle Symphony' with long vase life. Bull Natl Inst Flor Sci 2006, 5:1-16.

19. Tanase K, Onozaki T, Satoh S, Shibata M, Ichimura K: Differential expression levels of ethylene biosynthetic pathway genes during senescence of long-lived carnation cultivars. Postharvest Biol Tech 2008, 47:210-217.

20. Weber APM, Weber KL, Carr K, Wilkerson C, Ohlrogge JB: Sampling the Arabidopsis transcriptome with massively parallel pyrosequencing. Plant Physiol 2007, 144:32-42.

21. Yagi M, Kimura T, Yamamoto T, Isobe $\mathrm{S}$, Tabata S, Onozaki T: QTL analysis for resistance to bacterial wilt (Burkholderia caryophylli) in carnation (Dianthus caryophyllus) using an SSR-based genetic linkage map. Mol Breed 2011, 30:495-509

22. Novaes E, Drost DR, Farmerie WG, GJ Pappas Jr, Grattapaglia D, Sederoff RR, Kirst M: High-throughput gene and SNP discovery in Eucalyptus grandis, an uncharacterized genome. BMC Genomics 2008, 9:312.

23. Blanca J, Canizares J, Roig C, Ziarsolo P, Nuez F, Pico B: Transcriptome characterization and high throughput SSRs and SNPs discovery in Cucurbita pepo (Cucurbitaceae). BMC Genomics 2011, 12:104.

24. Barakat A, DiLoreto DS, Zhang Y, Smith C, Baier K, Powell WA, Wheeler $N$, Sederoff R, Carlson JE: Comparison of the transcriptomes of American chestnut (Castanea dentata) and Chinese chestnut (Castanea mollissima) in response to the chestnut blight infection. BMC Plant Biol 2009, 9:5.

25. Alagna F, D'Agostino N, Torchia L, Servili M, Rao R, Pietrella M, Giuliano G, Chiusano ML, Baldoni L, Perrotta G: Comparative 454 pyrosequencing of transcripts from two olive genotypes during fruit development. BMC Genomics 2009, 10:399. 
26. Vega-Arreguín JC, Ibarra-Laclette E, Jiménez-Moraila B, Martínez O, VielleCalzada JP, Herrera-Estrella L, Herrera-Estrella A: Deep sampling of the Palomero maize transcriptome by a high throughput strategy of pyrosequencing. BMC Genomics 2009, 10:299.

27. Guo S, Zheng Y, Joung J-G, Liu S, Zhang Z, Crasta OR, Sobral BW, Xu Y Huang S, Fei Z: Transcriptome sequencing and comparative analysis of cucumber flowers with different sex types. BMC Genomics 2010, 11:384.

28. Luo H, Li Y, Sun C, Wu Q, Song J, Sun Y, Steinmetz A, Chen S: Comparison of 454-ESTs from Huperzia serrata and Phlegmariurus carinatus reveals putative genes involved in lycopodium alkaloid biosynthesis and developmental regulation. BMC Plant Biol 2010, 10:209.

29. Newcomb RD, Crowhurst RN, Gleave AP, Rikkerink EHA, Allan AC, Beuning $L L$, Bowen $J H$, Gera E, Jamieson KR, Janssen BJ, Laing WA, McArtney S, Nain B, Ross GS, Snowden KC, Souleyre EJF, Walton EF, Yauk Y: Analyses of expressed sequence tags from apple. Plant Physiol 2006, 141:147-166.

30. Scovel G, Ben-Meir H, Ovadis M, Itzhaki H, Vainstein A: RAPD and RFLP markers tightly linked to the locus controlling carnation (Dianthus caryophyllus) flower type. Theor App/ Genet 1998, 96:117-122.

31. Kimura T, Yagi M, Nishitani C, Onozaki T, Ban Y, Yamamoto T: Development of SSR markers in carnation (Dianthus caryophyllus). J Japan Soc Hort Sci 2009, 78:115-123.

32. Smulders MJM, Rus-Kortekaas W, Vosman B: Microsatellite markers useful throughout the genus Dianthus. Genome 2000, 43:208-210.

33. Smulders MJM, Noordijk Y, Rus-Kortekaas W, Bredemeijer GMM, Vosman B: Microsatellite genotyping of carnation varieties. Theor Appl Genet 2003, 106:1191-1195

34. Yagi M, Onozaki T, Taneya M, Watanabe H, Yoshimura T, Yoshinari T, Ochiai $Y$, Shibata M: Construction of a genetic linkage map for the carnation by using RAPD and SSR markers and mapping quantitative trait loci (QTL) for resistance to bacterial wilt caused by Burkholderia caryophylli. J Japan Soc Hort Sci 2006, 75:166-172.

35. Rodríguez-Concepción M, Boronat A: Elucidation of the methylerythritol phosphate pathway for isoprenoid biosynthesis in bacteria and plastids. A metabolic milestone achieved through genomics. Plant Physiol 2002, 130:1079-1089.

36. Galpaz N, Ronen G, Khalfa Z, Zamir D, Hirschberg J: A chromoplast-specific carotenoid biosynthesis pathway is revealed by cloning of the tomato white-flower locus. Plant Cell 2006, 18:1947-1960.

37. Ohmiya A: Carotenoid cleavage dioxygenases and their apocarotenoid products in plants. Plant Biotechnol 2009, 26:351-358.

38. Tan B-C, Joseph LM, Deng W-T, Liu L, Li Q-B, Cline K, McCarty DR: Molecular characterization of the Arabidopsis 9-cis epoxycarotenoid dioxygenase gene family. Plant J 2003, 35v44-56.

39. Tanaka A, Tanaka R: Chlorophyll metabolism. Curr Opin Plant Biol 2006, 9248-255

40. Terahara N, Takeda K, Harborne JB, Self R, Yamaguchi M: Anthocyanins acylated with malic acid in Dianthus caryophyllus and D. deltoides. Phytochemistry 1986, 251715-1717.

41. Terahara N, Yamaguchi M: ${ }^{1} \mathrm{H}$ NMR spectral analysis of the malylated anthocyanins from Dianthus. Phytochemistry 1986, 25:2906-2907.

42. Bloor SJ: Blue flower colour derived from flavonol-anthocyanin copigmentation in Ceanothus papillosus. Phytochemistry 1997, 45:1399-1405.

43. Nakayama M, Koshioka M, Yoshida H, Kan Y, Fukui Y, Koike A, Yamaguchi M: Cyclic malyl anthocyanins in Dianthus caryophyllus. Phytochemistry 2000, 55:937-939.

44. Grotewold E: The genetics and biochemistry of floral pigments. Annu Rev Plant Biol 2006, 57:761-780.

45. Ogata J, Itoh Y, Ishida M, Yoshida H, Ozeki Y: Cloning and heterologous expression of a cDNA encoding flavonoid glucosyltransferase from Dianthus caryophyllus. Plant Biotechnol 2004, 21:367-375.

46. Matsuba Y, Sasaki N, Tera M, Okamura M, Abe Y, Okamoto E, Nakamura H,

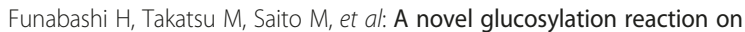
anthocyanins catalyzed by acyl-glucose-dependent glucosyltransferase in the petals of carnation and delphinium. Plant Cell 2010, 22:3374-3389.

47. Sakuta M, Ohmiya A: Pigment Biosynthesis II: Betacyanins and Carotenoids. In Plant Metabolism and Biotechnology. Edited by Ashihara $\mathrm{H}$, Crozier A, Komamine A.: John Wiley \& Sons, Ltd; 2011:343-372.

48. Abeles FB, Morgan PW, Saltveit ME Jr: Ethylene in Plant Biology. 2nd edition. New York: Academic Press; 1992.
49. Nichols R: Sites of ethylene production in pollinated and unpollinated senescing carnation (Dianthus caryophyllus) inflorescence. Planta 1977 135:155-159.

50. Kende H: Ethylene biosynthesis. Ann Rev Plant Physiol Plant Mol Biol 1993, 44:283-307.

51. Yang SF, Hoffman NE: Ethylene biosynthesis and its regulation in higher plants. Annu Rev Plant Physiol 1984, 35:155-189.

52. Hoeberichts FA, van Doorn WG, Vorst O, Hall RD, van Wordragen MF: Sucrose prevents up-regulation of senescence-associated genes in carnation petals. J Exp Bot 2007, 58:2873-2885.

53. Leverentz MK, Wagstaff C, Rogers HJ, Stead AD, Chanasut U, Silkowski H, Thomas B, Weichert H, Feussner I, Griffiths G: Characterization of a novel lipoxygenase-independent senescence mechanism in Alstroemeria peruviana floral tissue. Plant Physiol 2002, 130:273-283.

54. $\mathrm{Xu} Y$, Hanson MR: Programmed cell death during pollination-induced petal senescence in petunia. Plant Physiol 2000, 122:1323-1333.

55. Vrebalov J, Ruezinsky D, Padmanabhan V, White R, Medrano D, Drake R, Schuch W, Giovannoni J: A MADS-box gene necessary for fruit ripening at the tomato ripening-inhibitor (Rin) locus. Science 2002, 296:343-346.

56. Ito Y, Kitagawa M, Ihashi N, Yabe K, Kimbara J, Yasuda J, Ito H, Inakuma T, Hiroi S, Kasumi T: DNA-binding specificity, transcriptional activation potential, and the rin mutation effect for the tomato fruit-ripening regulator RIN. Plant J 2008, 55:212-223.

57. Lin Z, Hong Y, Yin M, Li C, Zhang K, Grierson1 D: A tomato HD-Zip homeobox protein, LeHB-1, plays an important role in floral organogenesis and ripening. Plant J 2008, 55:301-310.

58. Itkin M, Seybold H, Breitel D, Rogachev I, Meir S, Aharoni A: TOMATO AGAMOUS-LIKE 1 is a component of the fruit ripening regulatory network. Plant J 2009, 60:1081-1095.

59. Eveland AL, McCarty DR, Koch KE: Transcript profiling by 3 '-untranslated region sequencing resolves expression of gene families. Plant Physiol 2008, 146:32-44

60. Sato S, Isobe S, Asamizu E, Ohmido N, Kataoka R, Nakamura Y, Kaneko T, Sakurai N, Okumura K, Klimenko I, et al: Comprehensive structural analysis of the genome of red clover (Trifolium pratense L.). DNA Res 2005, $12: 301-364$

doi:10.1186/1471-2164-13-292

Cite this article as: Tanase et al.: Transcriptome analysis of carnation (Dianthus caryophyllus L.) based on next-generation sequencing technology. BMC Genomics 2012 13:292.

\section{Submit your next manuscript to BioMed Central and take full advantage of:}

- Convenient online submission

- Thorough peer review

- No space constraints or color figure charges

- Immediate publication on acceptance

- Inclusion in PubMed, CAS, Scopus and Google Scholar

- Research which is freely available for redistribution 